\title{
Efficacy of separate asthma therapy for particular phenotypes of asthma children
}

\author{
Alexey Perminov, Vladimir Petrov, Ivan Shishimorov, Olga Magnitskaya, Igor Nefedov \\ Clinical pharmacology, The Volgograd State Medical University, Russia
}

Introduction. Improving efficiency of basic asthma therapy is actual problem of asthma treatment now. Genotyping of asthma proved to be an ineffective method of solving this task. Asthma phenotype personolized treatment manner can be perspective for solutions to this problem. Mg defficiency may be one of the reasons of ineffective asthma treatment.

Methods. There were performed assesment of $\mathrm{Mg}$ defficiency $(<1,65 \mathrm{mmol} / \mathrm{L}$ in erythrosites) prevalence in asthma childern ( $n=141$ ). Patients ( $n=50$, middle age14,2 y.o., boys/girls 58/17) with and without Mg deficiency were stratifite by regime FLU/MON (low dose fluticasone propionate (inhalation) plus montelukast (per os), groups 1 and 3) or regime FLU/SAL (fixed combination of low dose fluticasone propionate plus salmeterol, groups 2 and 4). Main estimated parameters in a 3-month treatment were: assessment of asthma control level (GINA), rescue medication requirement, asthma exacerbation frequency.

Results. Mg deficiency prevalence were revealed in 41.1\% (58/141) asthma children in Volgograd. In a 12 weeks rescue medication consumption were equal in all groups of treatment. In groups without $\mathrm{Mg}$ deficiency asthma control level were $40 \%(4 / 10)$ vs 73,3\% (11/15) in a 12 weeks FLU/MON and FLU/SAL treatment respectively $(\mathrm{p}<0,05)$. In Mg deficiency groups asthma control level were $30 \%(3 / 10)$ vs $40 \%(6 / 15)$ in a 12 weeks FLU/MON and FLU/SAL treatment respectively. Asthma exacerbation frequency were $40 \%(6 / 15)$ vs $6,6 \%(1 / 15)$ respectively $(p<0,05)$ in FLU/SAL groups with and without Mg deficiency. Asthma exacerbation frequency were equal in both FLU/MON groups with and without Mg deficiency (20\% (2/10) vs 20\% (2/10) respectively).

Conclusion. Mg deficiency asthma children phenotype decreases FLU/SAL efficiency and do not influence to FLU/MON efficiency. 